\title{
Influence of Mechanical Tolerances on Field Quality in the LHC Main Dipoles
}

\author{
Walter Scandale, Ezio Todesco and Pacla Tropea \\ CERN, LHC Division, Geneva, Switzerland
}

\begin{abstract}
We evaluate the influence of mechanical tolerances on the field quality in the LHC dipoles. We show that the most relevant effect is due to tolerances on the coil and on tho internal part of the collars. The sensitivities of the field error multipoles on the mechanical tolerances are worked out using a finite element model of the dipole cross section. $\boldsymbol{A}$ MonteCarlo method is used to simulate the overall effect of both collar and coil tolerances on field quality. Correlation between random multipoles is worked out, and a comparison with the target table of the LHC field errors is given.
\end{abstract}

\section{INTRODUCTION}

The Large Hadron Collider (LHC) [1], under construction at CERN, uses superconducting magnets operating at a temperature of $1.9 \mathrm{~K}$ to guide the circulating particles. Protons are injected at low field in the magnets and circulate for several million tums before they are accelerated to the collision energy. Nonlinear fields eventually induced by magnet imperfections may produce uncontrollable instabilities in the particle trajectories, limiting the region of the stable motion, and thereby decreasing the accurnulated intensity and the luminosity of the collider. This phenomenon is particularly harnful during the injection plateau, when the beam size is maximum. There are three main sources of field imperfections in a superconducting magnet: the non-ideal geornetry of the coils, the persistent currents and the iron saturation. Here, we will discuss the consequences of the non-ideal geometry, induced by mechanical tolerances, in the specific case of the LHC main dipoles, Our analysis follows that of Refs $[2,4]$.

The nominal shape of the LHC dipole coils is such that significant systematic sextupole and decapole components are intentionally superimposed on the main bending field, with the aim of compensating the effect of persistent currents at injection. Coil deformations, induced by assembly prestress, themnal shrinkage during cool-down and mechanical tolerances, are potential sources of additional utwanted field errors, which must be evaluated and possibly controlled. The effect is expected to be visible on field-shape harmonics up to order $n=5$ and negligible above. It can be computed using the following procedure. The mechanical response of the dipole cross section is simulated with a finite element program, based on the code ANSYSTM [5]. The coil defornations induced by tolerances are used to compute the modified field-shape errors, with the program ROXIE [6]. MonteCarlo simulations are used to estimate statistically the intensity of the induced maltipoles. The maximum allowed tolerances for dipole series production are finally determined by imposing limitations on the unwanted multipoles. In Section II we describe the mechanical model of the dipole, in Section III we show how we introduce mechanical tolerances in the dipole model, in Section IV we discuss the effect of tolerances on collars and in Section V the effect on coils. In Section VI we present our final considerations.

\section{MECHANICAL MODEL OF THE DIPOLE}

The nominal cross section of the dipole cold mass contains two beam apertures in a common yoke, as shown in Fig. 1. Its shape is symmetric with respect to the horizontal and the vertical mid-planes. Each coil quadrant contains six blocks of conductors separated by four copper wedges.

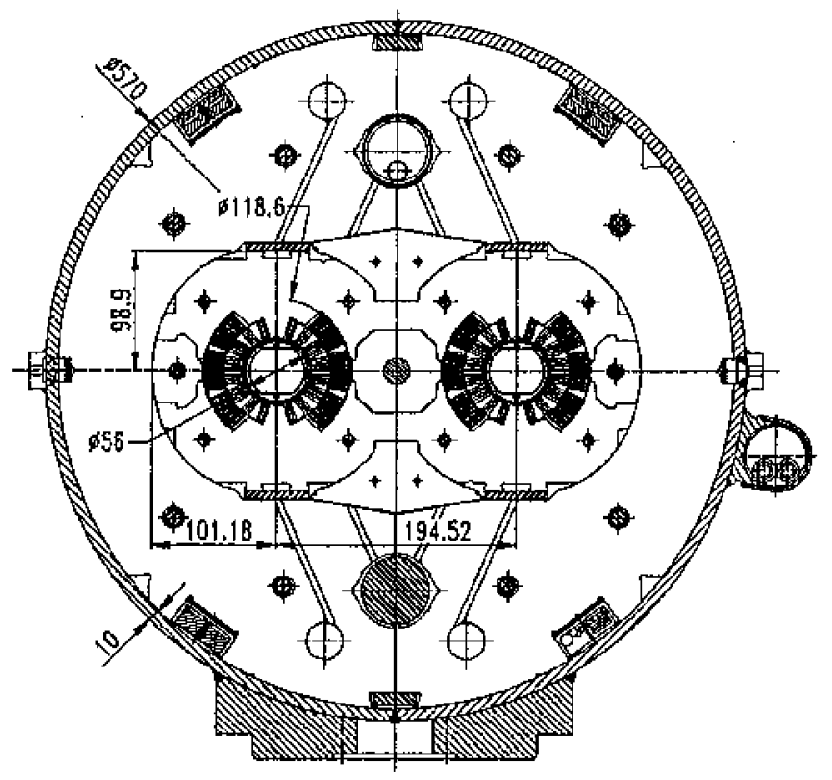

Fig. 1. Nominal cross-section of the LHC dipole.

A finite-element model of the dipole cross section is implemented in ANSYSTM, and used to evaluate the 2D deformations induced by assembly prestress, thermal slrinkage during the cool-down and mechatical tolerances, neglecting the effect of the magnet ends. The magnet geometry is subdivided into homogeneous regions with specific values of Young and Poisson moduli and thermal contraction coefficients. A model in which the two main components, i.e. the copper wedges and the superconducting blocks are well distinct describes the coil. The superconducting blocks are considered as homogeneous. Their properties are a weighed mixture of those of their main components, i.e. the superconducting cables and the polyimide insulation. As a result of that, the Young modulus of the blocks is not independent of the applied stress. The nominal shape of the coil cortesponds to a dipole temperature of $1.9 \mathrm{~K}$ and to a coil stress of $40 \mathrm{MPa}$. Its azirnuthal length at room temperature and in absence of stress is easily obtained with the ANSYS model itself. We introduce mechanical tolerances by acting on the contact elements between the collars and the coils, with the 
precautions described in Section III. The induced deformations are post-processed by a set of ad hoc developed prograns, called ANSIA, and passed to the input of the program ROXIE, which computes the magnetic field and the induced variation of the multipolar contents.

The present dipole model provides a first order estimate of the effect of tolerances on field-shape errors. Further work is in progress to have more precise experimental data on the mechanical and thermal properties of the coil, that will be implemented in our model.

\section{III, Model For MEchanical Tolerances.}

Our model contains only one quarter of the cold mass, i.e. one half of an aperture of two-in-one dipole cross-section. The coil design is such that in addition to the dipolarbending field one produces sextupole and decapole components to compensate the effect of persistent current at injection. These geometric components are indicated in Table 1 , up to $n=17$. The computed values are given in units of $10^{-4}$, at a reference radius $R_{\mathrm{F}}$ of $17 \mathrm{~mm}$. Odd normal harmonics are induced by the coil shape. Even normal harmonics are induced by the left-right asymmetry of the two-in-one collars. Skew components are zero, for symmetry reason.

TABLE 1

GECMOTRICAL MULTTPOIES COMPUTED AT NJJCTION FOR THE NOMINAL DIFOL.E CROSS SFCTION. UNITS OF $10^{-1}$ AT THE REFERENCE RADIUS $\mathrm{R}_{\mathrm{HEF}}=17 \mathrm{~mm}$.

\begin{tabular}{cc}
\hline Harmonic number & Normal componept \\
\hline 2 & -0.32 \\
3 & 6.25 \\
4 & 0.16 \\
5 & -0.90 \\
6 & 0.00 \\
7 & 0.63 \\
8 & 0.00 \\
9 & 0.10 \\
10 & 0.00 \\
11 & 0.59 \\
12 & 0.00 \\
13 & 0.07 \\
14 & 0.00 \\
15 & 0.03 \\
16 & 0.00 \\
17 & -0.05 \\
\hline
\end{tabular}

Boundary suffaces between collars and coils, modeled by contact elements, allow simulating mechanical tolerance. With our model we can only describe shape errors with an inherent up-down symmetry inducing only normal harmonics. In real life however, skew multipoles will also appear, because of the up-down tolerances, not yet included in our model. Nomal and skew harmonics are expected to have the same order of magnitude.

In principle, the deviation from nominal design of dipole components, like collars, copper wedges, or superconducting blocks, can be of any shape. In fact, the production process limits the possible shapes. Analyzing the measurements of the available components, we decided to use shape emors of the following type.

We assume that straight lines are preserved and only the end-points can be displaced, within tolerances, in the same direction (line shift), see Fig. 2a, or in opposite direction (line tilt), see Fig $2 \mathrm{~b}$.

When arcs of circumference are considered, we assume that the circular shape is preserved and that the are end-points can move, within tolerances, in the same direction (shift) or in the opposite direction (tilt).

The specified tolerances are assumed to represent $3 . \sigma$ of a Gaussian distribution, independently of the specific production features, not yet known in details. In a tilt or in a shift the end-points are displaced by the same amount in equal or in opposite directions respectively.

\section{TOLERANCES ON COLLARS}

We assume that mechanical tolerances may affect the geometry of the collars in the regions indicated in Fig. 3. Indeed, the errors of the external part of the collar have a negligible influence on coil deformations and will be disregarded in our following consideration. Instead, the shape errors of the intemal part of the collar are quite important, as discussed below. A shift or a tilt in that region may produce a sizeable change of the field-shape multipoles. An estinate of the sensitivity to this effect is given in Tables II and III, where the variations of the lowest order hamonics, from $b_{3}$ to $b_{6}$, are quoted for a shift or a tilt of $0.1 \mathrm{~mm}$, respectively, in the appropriate area.
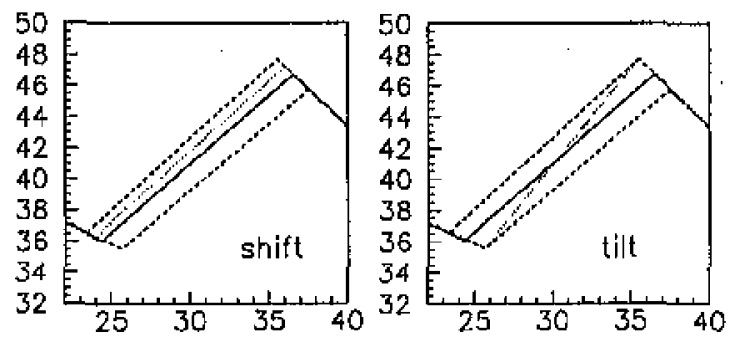

Fig 2a, Positive collar-shift.

Fig. 2b. Positive collar-tilt.

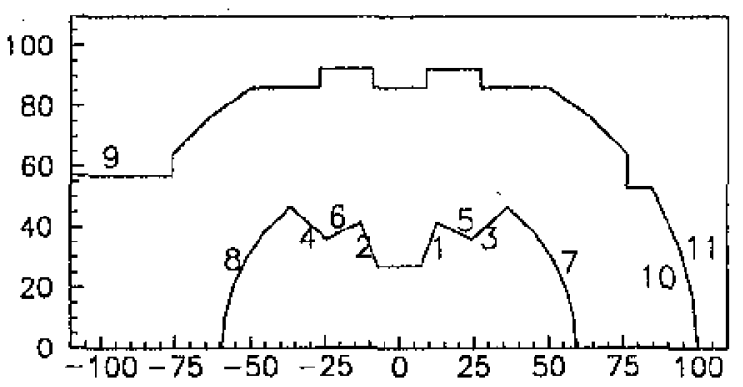

Fig. 3. Schematic representation of the one-half collar. The numbers indicate the area affected by mechanical tolerances. 
The induced multipoles vary almost linearly with the shape errors in the range $\pm 0.1 \mathrm{~mm}$, considered in our calculations. This is shown, for instance, in Fig 4, where the low order harmorics $b_{2}$ to $b_{5}$ are plotted as a function of the inner left collar-nose size (region 2). In the same Figure, we also show the difference of behavior between our model (solid lines) and a model (dotted lines) where all the effect of the tolerances is rigidly transmitted to the coil, without taking into account deformations.

TABLE II

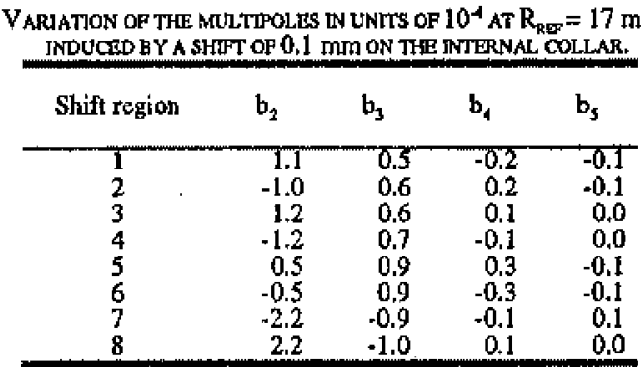

TABLE III

VARIATTON OF THE MUITIPOLES IN UNITS OF $10^{-4}$ AT $\mathrm{R}_{30 P}=17 \mathrm{~mm}$, INDUCED \& $Y$ A TILT OF $0.1 \mathrm{~mm}$ ON TER INELPAL COLAR

\begin{tabular}{crrrr} 
Tilt region & \multicolumn{1}{c}{$\mathbf{b}_{2}$} & \multicolumn{1}{c}{$\mathbf{b}_{3}$} & \multicolumn{1}{c}{$\mathbf{b}_{4}$} & \multicolumn{1}{c}{$\mathbf{b}_{5}$} \\
\hline $\mathbf{1}$ & -0.4 & -0.2 & 0.02 & 0.2 \\
2 & 0.4 & -0.2 & -0.03 & 0.2 \\
3 & 0.04 & 0.02 & -0.01 & 0.0 \\
4 & -0.06 & 0.04 & 0.01 & 0.0 \\
5 & 0.4 & 0.7 & 0.3 & 0.1 \\
6 & -0.4 & 0.7 & -0.3 & -0.1 \\
7 & -0.4 & -0.7 & -0.4 & -0.1 \\
8 & 0.5 & -0.7 & 0.4 & -0.2 \\
\hline
\end{tabular}

The previous results can be used to identify corrective actions on field quality, based on the use of shims in the collar nose or even in the collar cavity. However, this is not a trivial task, since one has to ayoid stress variations in excess of $\pm 5 \mathrm{MPa}_{\text {, }}$ to preserve the optimum stress window of the layers.

We use a MonteCarlo method to simulate the simultaneous action of all possible shape errors. We consider the following tolerances:

- $\pm 10 \mu \mathrm{m}$ on the nose (shapes 1, 2, 3 and 4 in Fig. 3)

- 0 to $40 \mu \mathrm{m}$ on the collar cavity (shapes $5,6,7,8$ )

- $\pm 20 \mu \mathrm{m}$ on the external collar (shapes 9, 10, 11)

For each shape we consider emors with a Gaussian distribution truncated at $3 \cdot \sigma$, where $\pm 3 \cdot \sigma$, covers the whole tolerance. We consider 100 realizations of the possible error configurations, generated by 100 differenl seeds, we apply them to our numerical modeI of the dipole and evaluate the statistical properties of the induced muttipoles shown in Table IV. The induced multipoles are within the specifications. In particular, the multipoles are at least an order of magnitude smaller than the target considered tolerable for the stability of the circulating beam in the LHC. This is particularly important, since other effects, like the random fluctuation of the persistent currents and of the iron magnetization, are not yet included.

The multipoles resulting from the MonteCarlo simulation are well approximated (within less that $50 \%$ discrepancy) by the quadratic sum of the multipoles induced by tolerances of the individual regions of the collar. In particular, the most sensitive area is the circular part of the cavity, i.e. the regions $5,6,7$ and 8 of Fig. 3.
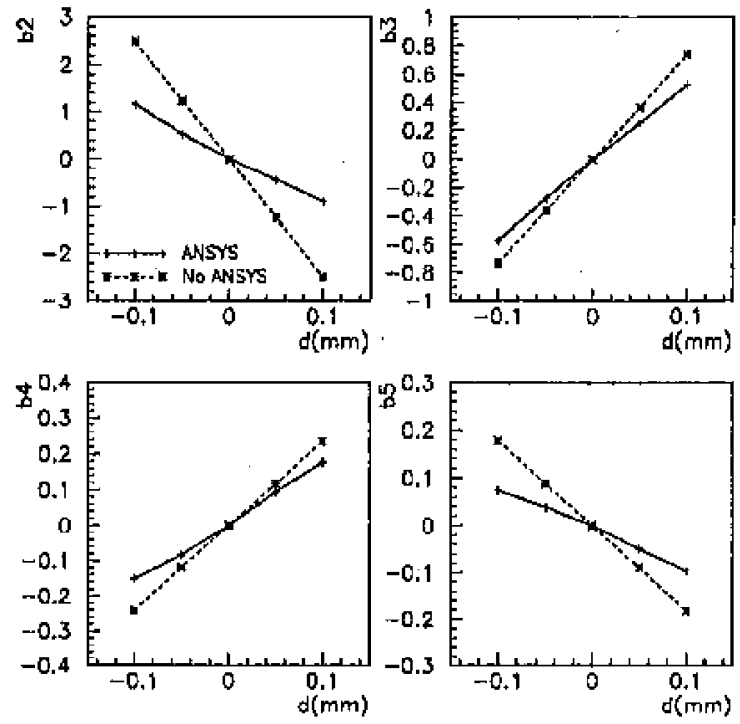

Fig. 4. Muttipoles versus shift of the inner left collar-nose (region 2).

TABLE IV

R.M.S. VARIATJON OP THE MULTPOLES IN UNTTS ON $10^{-1}$ AT $\mathrm{K}_{\text {gPF }}=17 \mathrm{~mm}$, ESTIMATED DY 100 SEEDS MONTECARLO SIMUL ATTON FOR COLLAR TOLERANCLS.

\begin{tabular}{lcccc}
\hline Shape-error & $b_{2}$ & $b_{3}$ & $b_{4}$ & $b_{5}$ \\
\hline $1,2,3,4$ & 0.06 & 0.03 & 0.01 & 0.00 \\
$5,6,7,8$ & 0.14 & 0.10 & 0.03 & 0.02 \\
$9,10,11$ & 0.01 & 0.02 & 0,00 & 0.00 \\
all tolerances & 0.16 & 0.16 & 0.04 & 0.03 \\
& & & & \\
Target values & - & 1.50 & 0.50 & 0.70 \\
\hline
\end{tabular}

The distributions of the geometric multipoles for the 100 seed MonteCarlo simulations are very close to a Gaussian distribution, centered around the nominal values. We also analysed conelations between hamonics of various orders. The correlation is strong between the allowed multipoles $b_{3}, b_{5}$ and $b_{7}$, and negligible between the other hasmonics.

The results of this Section have been used to re-cvaluate the tolerances for collar drawings. The initial requirements, used in our simulation, were very demanding and difficult and costly to be obtained in a consistent manner over mass 
production. The situation can be substantially improved by relaxing the collar tolerances. Scaling the results of Table IV, we can verify that there are no detrimental consequences on field quality when geometrical tolerances of regions 1 to 8 become $\pm 25 \mu \mathrm{m}$ and those of regions 9 to 11 become $\pm 40 \mu \mathrm{m}$.

\section{TOlerances ON COLls}

The tolerances on coil geometry are also described in parametric form, using contact elements in the ANSYS model. In this case, one has to take into account the component tolerarces and the coil curing process. The coil components are specified with the following characteristics:

- The azimuthal length of the copper wedges can be between 0 and $50 \mu \mathrm{m}$ longer than the nominal value.

- The thickness of each conductor can vary in the range \pm $6 \mu \mathrm{m}$; this imply that the inner coil length, containing 15 conductors, can vary by $\pm 90 \mu \mathrm{m}$, and the outer coil length, made by 25 conductors, can vary by $\pm 150 \mu \mathrm{m}$.

- The polyimide insulation has a thickness uncertainty of $\pm 5 \%$, inducing a total uncertainty of $\pm 180 \mu \mathrm{m}$ for the inner coil and of $\pm 300 \mu \mathrm{m}$ in the outer coil.

In fact, the tolerance of the azimuthal coil length after curing is only determined by the tolerances of the curing mold, since all the component uncertainties are smoothed down by the compression of the polyimide insulating film. We assume that the copper wedges preserve the original azimuthal length, since they are rather rigid. We also assume that the blocks are deformed proportionally to the number of conductors, until they recover the assumed value of the azimuthal coil length. In these conditions the uncertaitity of the insulating film and of the conductors are in practice, ineffective. We therefore assume the following tolerances for the cured coil:

- Azimuthal coil length: $\pm 20 \mu \mathrm{m}$.

- Azimuthal copper wedge length: 0 to $50 \mu \mathrm{m}$.

TABLE V

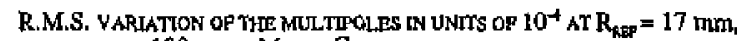
ESTRAATED EY 100 SERDS MONTECARLO SRMULATION FOR COLL TOLERANCES.

\begin{tabular}{lcccc} 
Shape-error & $\mathbf{b}_{2}$ & $\mathbf{b}_{3}$ & $\mathbf{b}_{4}$ & $\mathbf{b}_{3}$ \\
\hline Copper wedges & - & 0.21 & - & 0.05 \\
Coll length & - & 0.06 & - & 0.02 \\
All coil & - & 0.22 & - & 0.05 \\
All collar & 0.16 & 0.10 & 0.04 & 0.02 \\
Target values & - & 1.50 & 0.50 & 0.70 \\
\hline
\end{tabular}

In addition to that, we always assume a perfect four-fold symmetry in the coil shape for each dipole aperture, to simplify our simulations. Because of this testrictive hypothesis, we can study variations of the odd normal multipoles only.
Also in this case, we use MonteCarlo simulation to study 100 different coil realizations with shape errors within tolerances. 'The results are given in Tables V. The r.m.s variation of the multipoles is clearly within the target values presented in Section IV. In addition, because of the asymmetric tolerance of the copper wedge thickness, the average sextupole decreases by 0.7 units and the average decapole by 0.1 units at $R_{\mathrm{RFF}}=17 \mathrm{~mm}$. No correlation is found between the hamonics of different orders.

\section{CONCLUSIONS}

In this paper we analyzed the effect of mechanical tolerances on the field-shape of the LHC dipole. Simulations based on firite-element computer-model are used to estimate the mechanical defomation and stresses. A magnetostatic computer model accounts for field-shape errots induced by tolerances,

The main results are the following. The inner part of the collars and the coil are the most sensitive area of the LHC dipole. At present, their tolerance is chosen in a sound manner. The r.m.s, induced multipoles are one order of magnitude below the target values, and, at the same time, the expected variation of the coil prestres is not in excess of $\pm 6 \mathrm{MPa}$, a value still considered tolerable. Because of that, it is still possible to relax the mechanical tolerances of the collars, without detrimental consequences on field-shape, in order to simplify the collar series production and their quality control.

Moteover, we have identified areas where a shimming strategy can be deployed to recover possible errors during construction, i.e. the collar nose and the collat cavity. However, it is not obvious to modify by shimming a single multipole, in a selective manner, and to preserve at the same time the appropriate coil prestress.

We have also found that the multipole dependence on tolerances is still linear in the range of interest for the LHC dipole and that tolerances applied to different area of the collar or of the coil have independent effects that may be added quadratically.

\section{REFERENCES}

[1] The Large Hadron Collider, Conceptual Destgn, CERN/AC/95-05, 20 Oeiober 1995.

[2] R. Perin, CERN ATT195-60 (MA), Geneva (1996).

[3] R. Gupta, Particle Accelerators, vol. 55, pp 129-139, (1996).

[4] R Gupta, "Conocting field hasmonies after design in supereonducting magnets", $4^{\text {th }}$ IISSC, New Orleans, USA, (1992).

[5] ANSYSTM 5.4 Swanson Analysis Ine., Huston, PA, USA.

(6) S. Russenschuck, LHC Project Repart 159 (1997). 